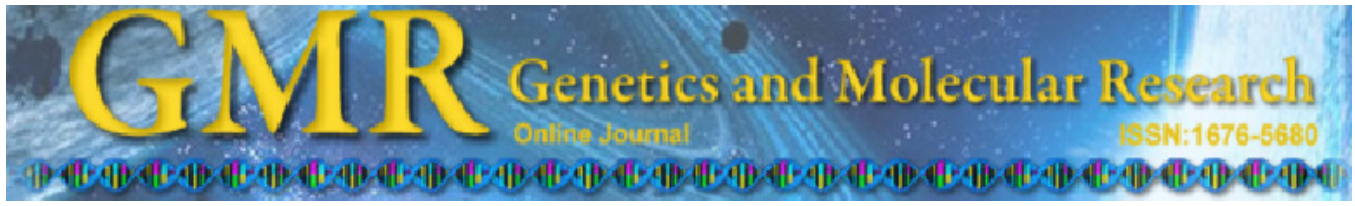

\title{
Nucleolar behavior during meiosis in four species of phyllostomid bats (Chiroptera, Mammalia)
}

M.R. Beguelini' ${ }^{1}$, S.R.C. Marchesin ${ }^{2}$, M.T.V. Azeredo-Oliveira ${ }^{1}$ and E. Morielle-Versute ${ }^{3}$

${ }^{1}$ Departamento de Biologia, Instituto de Biociências, Letras e Ciências Exatas, Universidade Estadual Paulista Julio de Mesquita Filho,

São José do Rio Preto, SP, Brasil

${ }^{2}$ Departamento de Biologia, Universidade Paulista,

São José do Rio Preto, SP, Brasil

${ }^{3}$ Departamento de Zoologia e Botânica, Instituto de Biociências, Letras e Ciências Exatas,

Universidade Estadual Paulista Julio de Mesquita Filho,

São José do Rio Preto, SP, Brasil

Corresponding author: E. Morielle-Versute

E-mail: morielle@ibilce.unesp.br

Genet. Mol. Res. 10 (2): 552-565 (2011)

Received September 13, 2010

Accepted November 5, 2010

Published April 5, 2011

DOI 10.4238/vol10-2gmr1060

\begin{abstract}
We analyzed the behavior of the nucleolus, nucleolar structures and nucleolus organizer regions (NORs) during meiotic division in four species of phyllostomid bats that have different numbers and locations of NORs. Nucleoli began disassembly at leptotene, and the subcomponents released from the nucleolus were dispersed in the nucleoplasm, associated with perichromosomal regions, or they remained associated with NORs throughout division. In Phyllostomus discolor, a delay in nucleolus disassembly was observed; it disassembled by the end of pachytene. The RNA complexes identified by acridine orange staining were observed dispersed in the nucleoplasm and associated with perichromosomal regions. FISH with rDNA probe revealed the number of NORs of the species: one NOR in Carollia per-
\end{abstract}


spicillata, one pair in Platyrrhinus lineatus and P. discolor, and three pairs in Artibeus lituratus. During pachytene, there was a temporary dissociation of the homologous NORs, which returned to pairing at diplotene. The variation in the number (from one to three pairs) and location of NORs (in sex or autosomal chromosomes, at terminal or interstitial regions) did not seem to interfere with the nucleolar behavior of the different species because no variation in nucleolar behavior that could be correlated with the variation in the number and chromosomal location of NORs was detected.

Key words: Nucleologenesis; Nucleolus; NORs; Chiroptera; Fluorescent in situ hybridization

\section{INTRODUCTION}

The compartmentalization of nuclear functions is, in part, a consequence of large complexes of machineries assembled in nuclear domains, where the biological functions associated with each have been the object of extensive investigation (Carmo-Fonseca et al., 2000; Louvet et al., 2005; Sirri et al., 2008). The nucleolus is the most distinctive nuclear sub-compartment in which ribosomal RNA (rRNA) biogenesis takes place. This process involves a series of events, including the transcription of rRNA genes, processing of the ribosomal pre-RNAs and association of pre-ribosomal particles (Raška et al., 2004, 2006a,b). Additionally, the relationship between the nucleolus and other nuclear bodies, such as the perinucleolar compartment and the Cajal body, raises the possibility that nucleoli have additional functions (Gall, 2001; Boisvert et al., 2007; Kotaja and Sassone-Corsi, 2007). The nucleolus has, in fact, been suggested to be a site for the biogenesis and/or maturation of other ribonucleoprotein machines, including the signal recognition particle, spliceosomal small nuclear ribonucleoproteins and telomerase (Mitchell et al., 1999; Olson et al., 2000; Politz et al., 2000; Andersen et al., 2005). The nucleolus has also been thought to participate in the processing or export of certain mRNAs and tRNAs and to be able to control the activities of specific regulatory factors via sequestration, as in the regulation of mitosis, cell cycle progression and proliferation, senescence, and stress responses (Visintin and Amon, 2000; Andersen et al., 2005; Boisvert et al., 2007; Sirri et al., 2008).

The dynamic integration of the different processes developed by the nucleolus generates a typical nucleolar organization. Three main specific components have been observed by electron microscopy in eukaryotic cells: the fibrillar centers (FC), observed as light areas surrounded by an area of high contrast; the dense fibrillar component (DFC), and the granular component (GC), where both the FCs and DFC are embedded. The primary rRNA transcripts are located at the junction of the FCs and DFC, while primary rRNA processing begins in the DFC and continues toward the GC during RNA migration (Hernandez-Verdun, 2006a,b).

During mitotic cell division, the contents of the nucleolus (e.g., RNA, proteins and enzymatic complexes) are dispersed to different parts of the cell. Generally, the nucleolus becomes disorganized in late prophase when ribosomal DNA (rDNA) transcription stops. Transcription is reactivated during mitotic anaphase (Leung et al., 2004; Sirri et al., 2008).

The ordinate nucleolus disorganization and the behavior of subcomponents can vary 
interspecifically. Some studies have reported the persistence of nucleolar material in mitotic cells, as indicated by the presence in early telophase of small silver grains and nucleolar masses designated as pre-nucleolar bodies (PNBs), which gradually disappear as nucleoli formed (Dundr et al., 2000; Savino et al., 2001). The functional significance of the presence of PNB in the cells remains unclear. It has been hypothesized that these bodies could represent a source of nucleolar RNA for the daughter cells while the new nucleolus is being organized or serve as an assembly platform for processing complexes in this period of the cell cycle (Dundr et al., 2000; Hernandez-Verdum, 2006b).

Few studies of the nucleolar cycle have been carried out during meiotic cell division in mammals, and observations have shown that in different species of rodents a nucleolar fragmentation occurs at the beginning of spermatogenesis and that a fraction of this nucleolar material migrates to the cytoplasm, where a specific structure is formed, known as the "chromatoid body", which apparently participates in some parts of the rodent spermiogenesis process (Paniagua et al., 1986; Peruquetti et al., 2008, 2010). The majority of the authors, however, have not reached a consensus about what happens to the nucleolus during meiotic cell division.

Thus, the objective of the present research was to study the nucleolus and the nucleolar disruption in meiotic cells of four species of bats that show a different number of nucleolus organizer regions (NORs) by silver nitrate and acridine orange staining and fluorescent in situ hybridization (FISH) with rDNA probe.

\section{MATERIAL AND METHODS}

\section{Specimens}

Investigations were carried out on two sexually mature specimens of Artibeus lituratus, Carollia perspicillata, Phyllostomus discolor, and Platyrrhinus lineatus. The animals were collected in the northwest of São Paulo State, Brazil (49W 22'45" 20S 49' 11"), and the specimens are deposited in the Chiroptera collection at the São Paulo State University (UNESP-IBILCE).

\section{Cell suspension and staining procedures}

The testes were removed and softened on Petri plates containing $\mathrm{KCl}(0.075 \mathrm{M})$ and colchicine $(0.1 \%)$ solution, for $40 \mathrm{~min}$ at room temperature. The cell suspension was centrifuged at $1000 \mathrm{rpm}$ for 6 min and the cells fixed in methanol:acetic acid (3:1). Freshly prepared slides were submitted to silver nitrate (Kavalco and Pazza, 2004) and acridine orange staining (Abrams et al., 1993), and FISH (Viegas-Péquignot, 1992) using as probe a recombinant plasmid (HM123) containing a fragment of Xenopus laevis 28S rDNA, biotinlabeled by nick-translation reaction. The slides with the different staining were analyzed in a light and fluorescence microscope (Axioskop 2 - Zeiss) fitted with an image analyzer. Cell images were captured with a CCD camera using the AxioVision 3.1 for Windows software.

\section{RESULTS}

\section{Silver nitrate staining}

Cell analysis of the four species subjected to silver impregnation revealed that, except 
for C. perspicillata, the number of nucleoli in spermatogonia in interphase varied. In C. perspicillata, only one nucleolus was observed (Figure 1A), whereas in P. lineatus (Figure 1B) and $P$. discolor (Figure 1C) one or two nucleoli were observed, and in A. lituratus (Figure 1D), one to six nucleoli, where three to five nucleoli were the most frequent numbers.

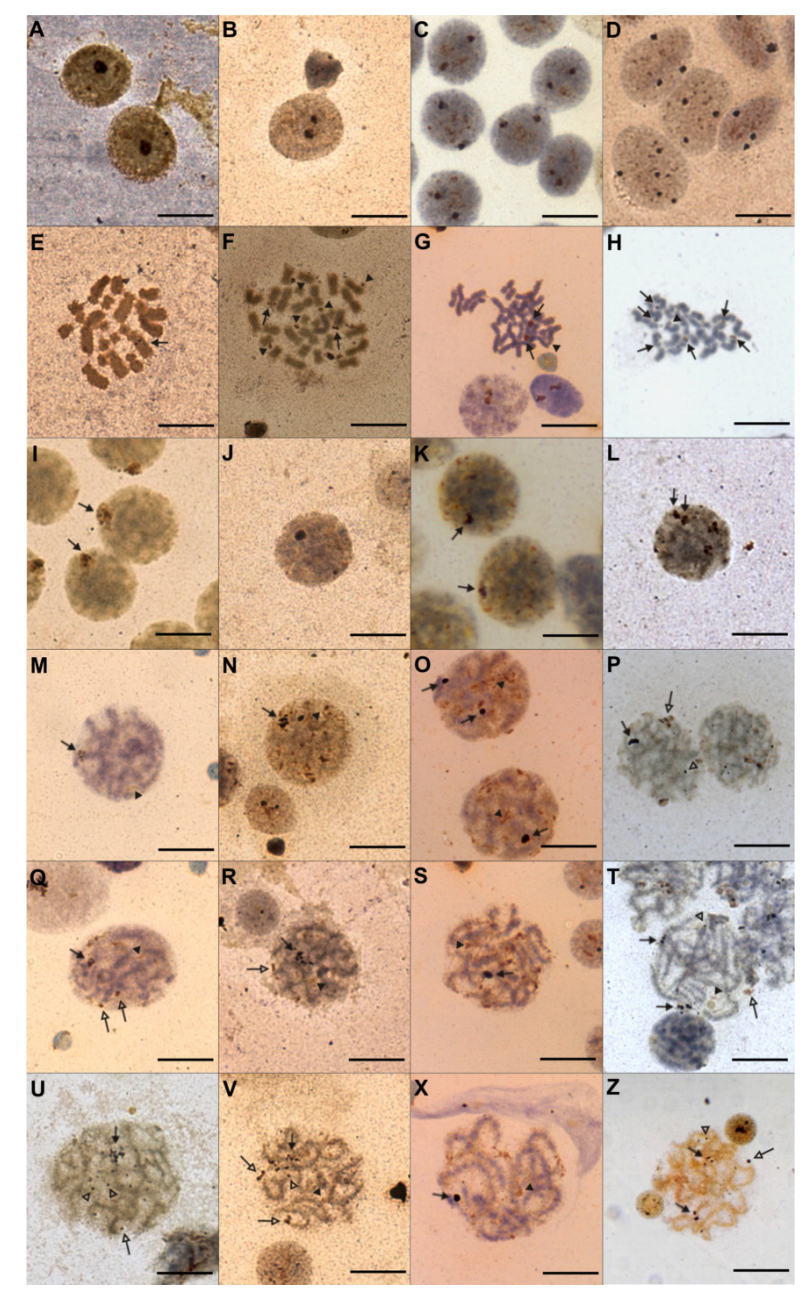

Figure 1. Testicular cells of Carollia perspicillata (first column), Platyrrhinus lineatus (second column), Phyllostomus discolor (third column), and Artibeus lituratus (last column) silver-stained. A-D. Spermatogonia in interphase with one nucleolus (A), two nucleoli (B,C) and three to five nucleoli (D). E. Spermatogonial mitosis. Note the interstitial nucleolus organizer region (NOR) in the long arm of the X-chromosome (arrow). F. Spermatogonial mitosis. Note the terminal NORs in one pair of chromosomes (arrows) and the persistence of nucleolar material (arrowheads). G. Spermatogonial mitosis. Note the staining of the two chromosomes carrying the NORs (arrows) and the persistence of nucleolar material (arrowhead). H. Spermatogonial mitosis. Note the staining of three pairs of chromosomes with NORs (arrows) and the persistence of nucleolar material (arrowhead). Primary spermatocytes at leptotene (I$\mathbf{L})$, zygotene (M-P), early pachytene (Q-T), and pachytene (U-Z) stages. Note the site of disruption of nucleolus (arrows), the material on the periphery of the nucleus (open arrows), the material dispersed in the nucleoplasm (arrowheads), and the material associated with perichromosomal regions (open arrowheads). Bars: $10 \mu \mathrm{M}$. 
Similar to that observed in interphase, the spermatogonia in mitosis showed variation in the number of chromosomes stained by silver nitrate. In C. perspicillata, which showed 21 chromosomes $(2 \mathrm{n}=21)$, only one chromosome, the X-chromosome, appeared silver-stained at the interstitial region, the NOR location (Figure 1E). In P. lineatus and $P$. discolor, whose cells showed $30(2 \mathrm{n}=30)$ and 32 chromosomes $(2 \mathrm{n}=32)$, respectively, one pair of chromosomes appeared labeled (stained) by silver nitrate in the terminal region, which are the carriers of the NORs. These are represented by two medium chromosomes in P. lineatus (Figure 1F) and two small chromosomes in $P$. discolor (Figure 1G). The largest number of chromosomes silver-stained was observed in spermatogonia of $A$. lituratus, which showed one to six chromosomes stained, representing the three pairs of chromosome carriers of terminal NORs (Figure 1H). In the three species, the presence of nucleolar fragments between the chromosomes was noted (Figure 1F-H, arrowheads).

In leptotene spermatocytes, the number of nucleoli followed the pattern exhibited by spermatogonia, i.e., one nucleolus in the cells of $C$. perspicillata (Figure 1I), one or two in P. lineatus (Figure 1J) and P. discolor (Figure 1K) and one to six in A. lituratus (Figure 1L), where the presence of three or four nucleoli was more frequent.

With the increase in chromosome condensation, the nucleolus began to disassemble, and the nucleolar components were observed in different locations of the cell. Some components appeared as silver-impregnated bodies spread in the nucleoplasm (Figures $1 \mathrm{M}-\mathrm{Z}$ and 2A-G, arrowheads) or displaced to the border of the nucleus (Figures $1 \mathrm{P}-\mathrm{Z}$ and $2 \mathrm{~A}-\mathrm{D}$, open arrows). These appeared at the end of leptotene, decreased in size during division, disappeared by diplotene, and were associated with nonspecific perichromosomal regions (Figures 1P-Z and 2A-D, open arrowheads), which appeared in zygotene and disappeared by diplotene. Another fraction was observed associated with the NOR throughout meiosis (Figures 1I-Z, arrow, and 2E-N). These characteristics were observed in three of the four species, i.e., C. perspicillata, P. lineatus and A. lituratus.

In $P$. discolor, despite that the sequence of nucleolar disassembly had been similar to other species, the nucleoli of the spermatocytes remained partially organized during most of meiotic division. The body with nucleolar material strongly silver-stained was observed from the leptotene stage (Figure 1K) to the late pachytene stage (Figure 1X), and it was only fragmented later, at the end of pachytene (Figure 2G). Despite the delay, the separated and fragmented material spread through the cytoplasm (Figure $2 \mathrm{G}$ ), and during diakinesis, only the nucleolar material that remained associated with the NORs was observed in the bivalents (Figure 2K).

In meiotic metaphase I, silver staining was restricted to NORs, where in C. perspicillata, only the interstitial labeling of the X-chromosome was observed (Figure 2I), in $P$. lineatus and $P$. discolor one or two chromosomes with NORs labeled (Figure $2 \mathrm{~N}$ and $\mathrm{O}$, respectively) and in A. lituratus, one to six chromosomes labeled by silver nitrate (Figure 2P). In meiotic metaphase II, two situations were recorded for $C$. perspicillata, the first showing 10 chromosomes, with the X-chromosome labeled (Figure 2M) and the second showing 11 chromosomes, without the presence of labeled chromosomes (Figure 2Q). In P. lineatus and P. discolor, one chromosome appeared labeled (Figure 2R and $\mathrm{S}$, respectively), whereas in $A$. lituratus, one to three chromosomes were observed with labeling (Figure 2T).

A single and very large nucleolus was observed in early spermatids of the species $C$. perspicillata (Figure 2U), P. lineatus (Figure 2V) and P. discolor (Figure 2X). In A. lituratus, the number of nucleoli ranged from one to three (Figure 2Z), although some cells showed more than three nucleoli. The nucleolus was disrupted with the entry of spermatids in the process of spermiogenesis, and no nucleolar material was observed in the later stages. 


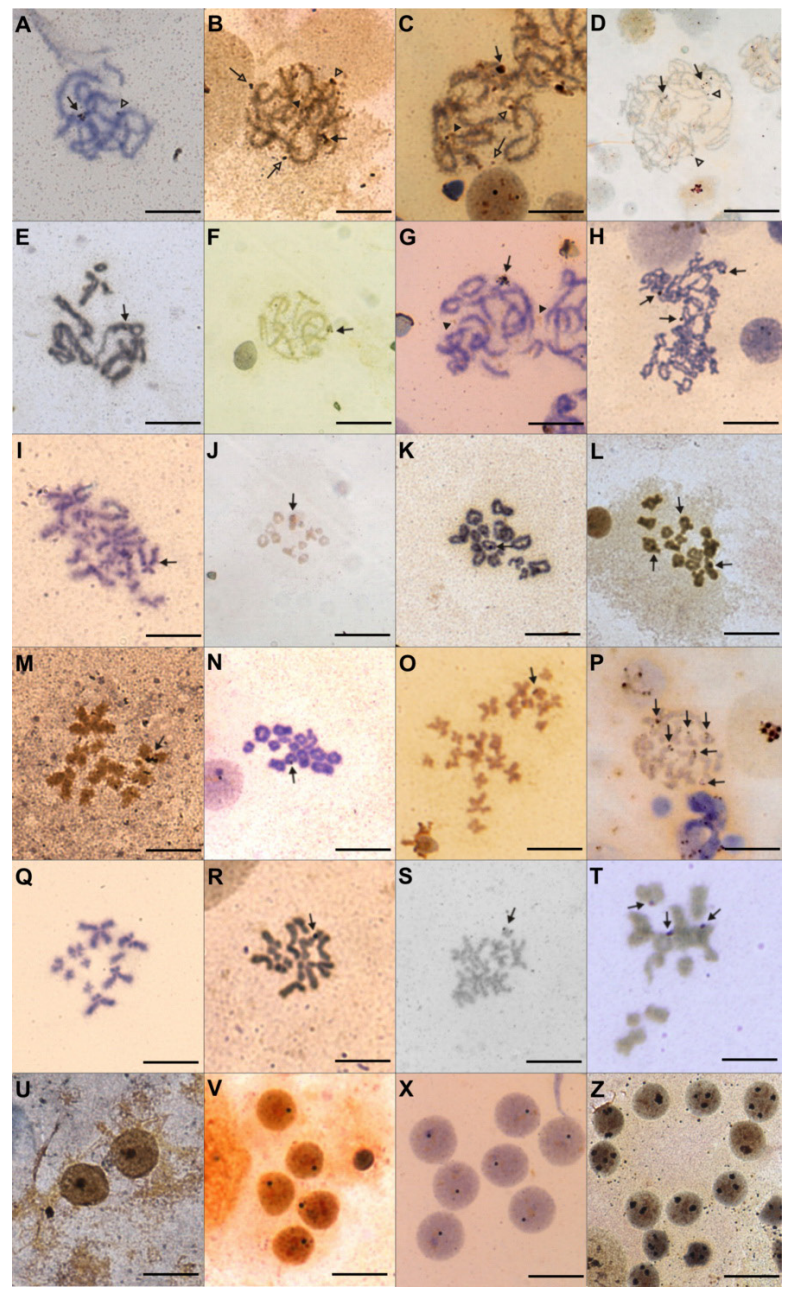

Figure 2. Testicular cells of Carollia perspicillata (first column), Platyrrhinus lineatus (second column), Phyllostomus discolor (third column), and Artibeus lituratus (last column) silver-stained. Primary spermatocytes in late pachytene stages (A-D). Note the site of disruption of nucleolus (arrows), the material moved to the periphery of nucleous (open arrows), the material dissolved in the nucleoplasm (arrowheads), and the material associated with perichromosomal regions (open arrowheads). E-G. Primary spermatocytes at diplotene. Note the staining of the trivalent- $X Y_{1} Y_{2}(\mathbf{E}-$ arrow) and the silver grains only in one region in $\mathbf{F}$ (arrow), and in one region (arrow) and dispersed in the nucleoplasm (arrowheads) in G. H. Primary spermatocytes in late pachytene, showing three labelings (arrows). I. Meiotic metaphase I with the labeling of the X-chromosome (arrow). J. Diakinesis bivalents without chiasm, showing labeling (arrow). K. Diakinesis showing labeling of ring bivalent (arrow). L. Diakinesis showing labeling of three bivalents (arrows). M. Meiotic metaphase II with 10 chromosomes and labeling on X-chromosome (arrow). N. Diakinesis showing labeling of ring bivalents (arrow). O. Meiotic metaphase I with one chromosome labeled (arrow). P. Meiotic metaphase I showing six labeled chromosomes (arrows). Q. Meiotic metaphase II with 11 chromosomes and without labeling. R. Meiotic metaphase II with a chromosome labeled (arrow). S. Meiotic metaphase II with a chromosome labeled (arrow). T. Meiotic metaphase II with three chromosomes labeled (arrows). U. Spermatids with a single nucleolus. V. Spermatids with a single nucleolus. X. Spermatids with a single nucleolus. Z. Spermatids with one to four nucleoli. Bars: $10 \mu \mathrm{M}$. 


\section{Acridine orange}

Acridine orange was used to differentiate DNA and nuclear RNA during meiotic division. When intercalated in DNA, it fluoresces green/yellow, and when associated with RNA it fluoresces red. The pattern of variation in acridine orange staining in preparations of the four species was similar. In early leptotene spermatocytes, red fluorescence was observed mainly concentrated in a single region, while lower fluorescence signals appeared dispersed throughout the nucleoplasm (Figure 3A-D, arrows). With the increase in chromosome condensation, fluorescence was observed in intimate association with the chromosomes, around the chromosomes and dispersed throughout the nucleoplasm (Figure 3D, N, P, and R). This fluorescence disappears in metaphase (Figure 3U-Z). In P. discolor, cells in late pachytene fluoresced more than in other species (Figure 3S), but it disappeared in metaphase (Figure 3X).

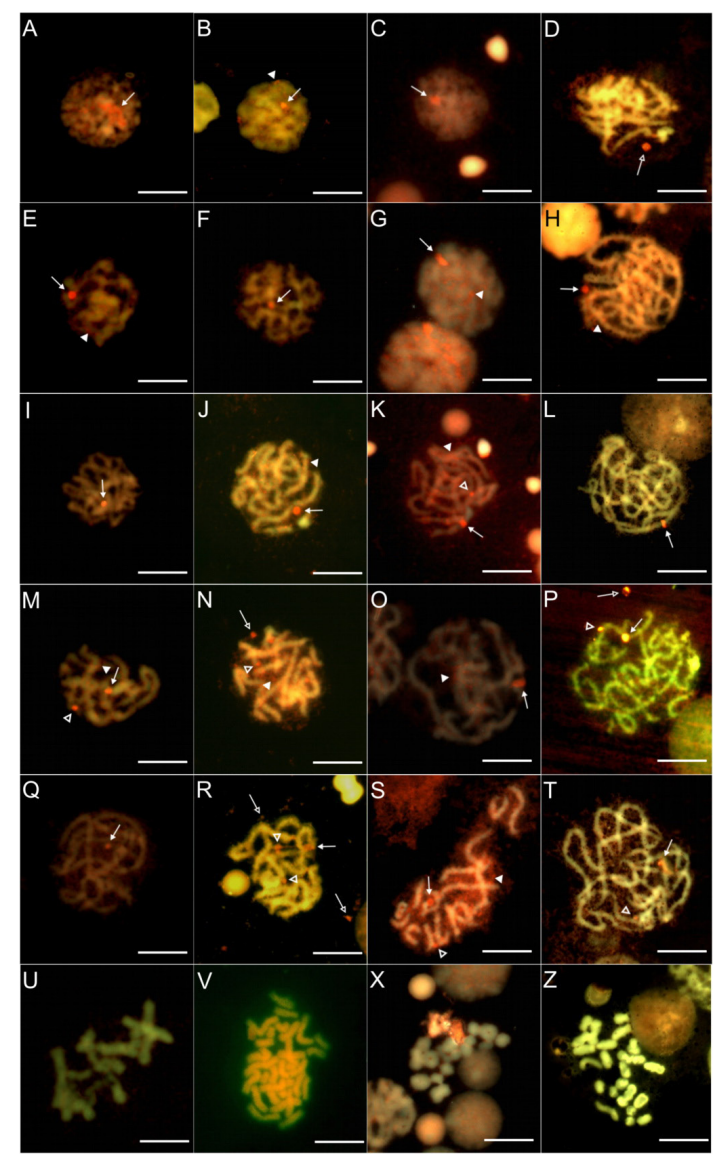

Figure 3. Testicular cells of Carollia perspicillata (first column), Platyrrhinus lineatus (second column), Phyllostomus discolor (third column), and Artibeus lituratus (last column) acridine orange-stained. Primary spermatocytes at leptotene (A-D), zygotene (E-H), early pachytene (I-L), pachytene (M-P), and late pachytene (Q-T) stages. Note the material associated with the NORs (arrows), dispersed in the nucleoplasm (arrowheads), associated with perichromosomal regions (open arrowheads), and the material that displaced from the nucleus (open arrows). Metaphases (U-Z). Note the absence of fluorescent staining, indicative of RNA. Bars: $10 \mu \mathrm{M}$. 


\section{Fluorescent in situ hybridization}

FISH with rDNA probe located the sequences and the number of rDNA sites in the species analyzed. In C. perspicillata, the spermatogonia and primary spermatocytes as well as the newly formed spermatids showed a single fluorescent signal, which varied little in size (or intensity) (Figure 4, first column).

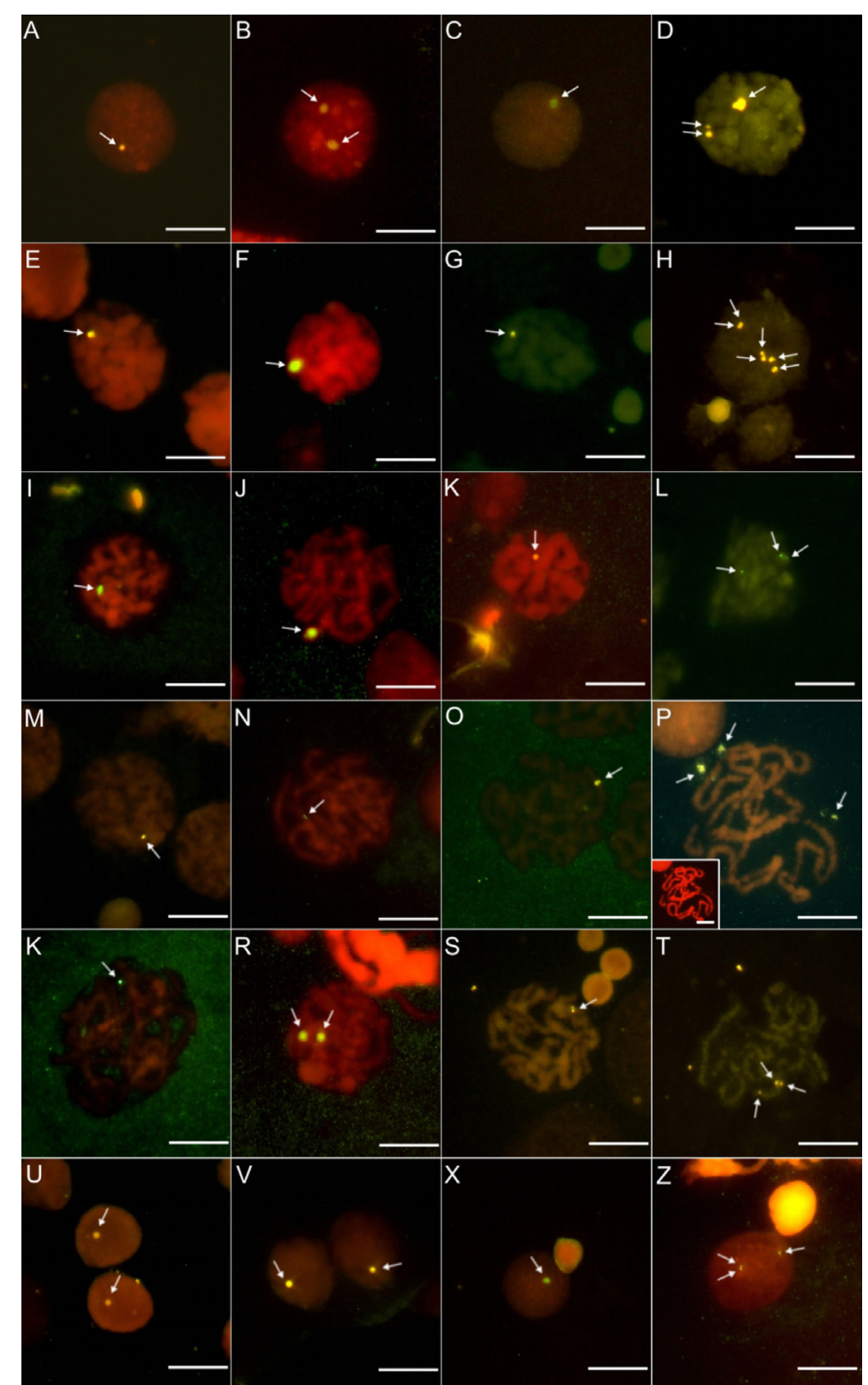

Figure 4. Testicular cells of Carollia perspicillata (first column), Platyrrhinus lineatus (second column), Phyllostomus discolor (third column), and Artibeus lituratus (last column) after fluorescent in situ hybridization with rDNA probe. A-C. Spermatogonia. D-G. Leptotene spermatocytes. H-L. Zygotene spermatocytes. M-T. Pachytene spermatocytes. U-Z. Spermatids. (Arrows, nucleolar organizer regions; Insert in $\mathbf{P}$, same propidium iodide-stained cell). Bars: $10 \mu \mathrm{M}$. 
In preparations of $P$. lineatus and $P$. discolor, the spermatogonia showed one or two fluorescent signals (Figure 4B and C, respectively), whereas in primary spermatocytes, most cells exhibited a single fluorescent signal (Figure $4 \mathrm{~F}$ and $\mathrm{J}$ and $4 \mathrm{G}$ and $\mathrm{K}$, respectively). However, in some cells in pachytene, the NORs were observed individually, showing two fluorescent signals (Figure $4 \mathrm{~N}$ and $\mathrm{R}$ and $4 \mathrm{O}$ and $\mathrm{S}$, respectively), unlike in the cells in late pachytene, diplotene and diakinesis, which displayed only one signal. In spermatids, only one signal was visualized (Figure $4 \mathrm{~V}$ and $\mathrm{X}$, respectively).

In the species A. lituratus, the primary spermatocytes in leptotene had two or three larger fluorescent signals (Figure 4D) or up to six smaller signals in zygotene (Figure 4H). These signals remained paired throughout division (Figure 4, last column). Spermatids exhibited more often one single, large fluorescent signal, or two or three smaller fluorescent signals (Figure 4Z).

\section{DISCUSSION}

\section{Interphase nucleoli}

Variations in number, size and shape of nucleoli observed in steady-state by microscopic methods are reflections of the dynamic processes to which the nucleoli are subjected. Therefore, the morphology is directly linked to their functional status, and they may also be influenced by or react to external stimuli (Politz et al., 2003; Raška et al., 2004; Louvet et al., 2005).

Similar to that observed by other authors who analyzed the mitotic cells of these species, in the present study, the number and size of nucleoli in spermatogonia and spermatids varied, and the maximum number of nucleoli observed corresponded to the number of NORs of species. This variation observed in meiotic cells can be attributed, similar to that observed in mitotic cells, mainly due to differential activation of NORs and nucleolar fusion event, the latter occurring at high frequencies in somatic cells of Chiroptera (Volleth, 1987; Morielle and Varella-Garcia, 1988; Souza and Araújo, 1990). In the species analyzed, nucleolar fusion was more evident in cells of $A$. lituratus, the species that had the largest number of NORs.

\section{Nucleolar subcomponents during meiotic division}

In spite of abundant experimental data on the dynamics of the nucleolus and its major proteins during mitosis, information on nucleolar dynamics during meiosis of vertebrates is scarce. It has been assumed that in higher eukaryotes, when the cells proceed through the phases of mitosis, pol I transcription gradually decreases until it is completely silenced and the nucleoli disperse and disappear. Thus, by the end of prophase, the nucleolus is no longer visible (Leung and Lamond, 2003; Dimario, 2004; Olson and Dundr, 2005; HernandezVerdun, 2005, 2006b; Sirri et al., 2008). The nucleolar subcomponents (proteins, RNAs and enzyme complexes) that dissociated from the nucleolus disperse to different parts of the cell, only coming together again in telophase when transcription is reactivated and when the new nucleolus is organized (Olson and Dundr, 2005).

In this study, although the species analyzed represent four different genera of Chiroptera, the nucleolar behavior during different stages of meiosis was similar in three of the four species, i.e., C. perspicillata, P. lineatus, and A. lituratus demonstrating that there was no interference of the taxon in nucleolar behavior. 
Similar results had been observed by Hofgärtner et al. (1979) by analyzing the pattern of activity of the nucleolus organizer in spermatogenesis in four species of mammals (Homo sapiens, Mus musculus, Rattus norvegicus, and Cavia cobaya) and by Schmid et al. (1982) who analyzed ten species of non-mammalian vertebrates, where both reported a very similar pattern of NOR activity in the various stages of spermatogenesis. Interspecific differences in the pattern of nucleolar activity occur in more distantly related taxa, when comparing, for example, species of mammals, insects and plants.

The disorganization of the nucleolus in the three species of bats began in leptotene, and with the advance in stages the nucleolar material released occupied different regions of the cell, i.e., part spread throughout the nucleoplasm, part became associated with the chromosomes in the form of a sheath, and a part remained associated with the NORs.

The only species whose cells showed a delay in nucleolus disorganization was $P$. discolor, where the nucleoli remained active and partly organized until the pachytene stage, from which followed the same pattern of relocation of nucleolar material observed in the other three species. This delay in nucleolar disassembly was interpreted as resulting from a greater application of synthesis by nucleoli of these cells to serve the specific physiological demands of individuals.

Despite the similarities observed in the pattern of NOR activity in the species analyzed by Hofgärtner et al. (1979) and Schmid et al. (1982), and in the species of bats examined, these differed from others with regard to the conditions observed in the stage of meiotic metaphase I and II. Both authors reported the presence of labelings associated with NORs only in spermatogonia up to the pachytene stage of meiotic prophase, and that during metaphase I and II and interkinesis, the silver staining disappeared completely. In the species of bats examined, silver staining was present on chromosomes with NOR organizers throughout the cell cycle.

The pattern of disorganization, distribution and relocation of nucleolar components observed in meiotic cells of the bats was interpreted as being similar to that observed for mitotic cells of higher eukaryotes including different species of vertebrates. Based on the analysis of proteins and nucleolar activity, several authors hypothesized that by suppressing the synthesis of rDNA in late prophase, transcription factors such as UBF, SL1 and topo I and several pol I subunits remain associated with the mitotic NORs, and the remaining nucleolar antigens (pol I subunits, rRNA processing and ribosome assembly components) move from the DFC and GC out of the nucleolus, toward the nuclear envelope along defined paths within the nucleoplasm. By metaphase, the antigens have reaccumulated in the perichromosomal sheath (Leung et al., 2004; Sirri et al., 2008).

The association of nucleolar proteins (fibrilarin, and B23 and Nop52) with the chromosome periphery is concurrent with chromosome condensation (Leung et al., 2004). It has been suggested that the association of these proteins with chromosomes ensures the equal distribution to daughter cells, allowing the reorganization of the nucleolus after mitosis.

In the present study, the stainings in perichromosomal regions observed in the cells of the species analyzed in zygotene and that disappeared by the diplotene were interpreted as remnants of material that compose the perichromosomal sheath.

In anaphase, when rDNA transcription is reactivated, the proteins associated with the perichromosomal regions and the transcribed snoRNAs and partially processed pre-rRNA detached from the chromosome periphery, moving to the cytoplasm where they associated with larger particles called "nucleolus-derived foci" (NDF) (Dundr et al., 2000; Olson et al., 2000). As the cells proceded through telophase, the number of NDFs decreased and other particles, 
the PNB, appeared in the cell. These subsequently fuse with the chromosomal NORs of the mitotic telophase and earlier interphase cells (Dundr et al., 2000).

Unlike that observed in meiotic cells of other animal species, such as different heteropteran species, where in metaphase I cells a prominent nucleolar mass was observed in the cell periphery and small silver grains were detected in metaphase II, indicating a nucleolar persistence in meiosis (Cattani and Papeschi, 2004; Costa et al., 2008; Morielle-Souza and Azeredo-Oliveira, 2008), in the present study, by metaphase the only nucleolar material observed in the meiotic cells was restricted to remains associated with the NORs. Nucleolar material between chromosomes was observed only in the mitotic metaphase. The presence in insects of the semi-persistent nucleolus has been attributed to a continuous biosynthetic activity required for spermiogenesis, which could be related to differences in the environmental, and therefore, physiological conditions of the individuals.

Despite that NORs labeled in meiotic metaphasic chromosomes have a smaller size compared to mitotic labeling, the amount of nucleolar material that remains associated with NORs during meiosis may be less than in mitosis, a fact that may be associated with differences in nucleolar dynamics in different phases of the cell cycle of these cells. The presence of nucleolar remnants in NORs could be important for the spermiogenesis process of bats, since unlike in insects nucleolar persistence has not been identified. Moreover, this material associated with chromosomes and NORs will be distributed between the two daughter cells, ensuring a subsequent reorganization of the nucleolus in the nucleus of spermatid, since it is not governed solely by the resumption of rDNA transcription (Sirri et al., 2008).

Studies involving the nucleolar cycle during spermatogenesis of the Mongolian gerbil and other vertebrate species have demonstrated nucleolar fragmentation and the probable migration of these nucleolar ribonucleoproteins to the cytoplasm, where these proteins seem to contribute to the chemical composition of the chromatoid body (Peruquetti et al., 2008). The migration of nucleolar material to the cytoplasm can then explain the faint staining of NORs in mammalian meiotic cells.

The disassembly and distribution of protein complexes and RNA described for nucleolar subcomponents of the cells of bats after silver staining was confirmed by acridine orange, which identified the presence of nucleolar RNA with a portion of the material dispersed in the nucleoplasm and associated with chromosomes. No signal was observed in metaphase chromosomes. These results corroborate the findings of Dundr et al. (2000) and Olson et al. (2000), who observed the presence of RNAs in NDFs and in the perichromosomal sheath and its absence in the material that is associated with NORs during metaphase.

The results of the present study provided evidence that the NORs reassume their activity in newly formed spermatids shortly after the two meiotic divisions, organizing a new functionally active nucleolus, which was clearly individualized by staining with silver nitrate and acridine orange.

\section{Nucleolus organizer regions and nucleolar behavior}

Bats show a wide variation in number and location of NORs, even in closely related taxa. This variation ranges from one pair of NORs as observed in Diaemus youngii and Desmodus rotundus, up to five pairs in different species of Myotis (Volleth 1987; Morielle and Varella-Garcia, 1988; Souza and Araújo, 1990; Baker et al., 1992; Morielle-Versute et al., 
1996). Moreover, the NORs occur both in terminal regions and interstitial regions of chromosome arms, and they generally correspond to the secondary constrictions.

The results of this study, especially those obtained by FISH corroborated the previous results obtained by Morielle and Varella-Garcia (1988) and Souza and Araújo (1990) for these same species by silver staining. FISH with rDNA probe confirmed the number and location of NORs on the X-chromosome of $C$. perspicillata, in the terminal region of a pair of autosomes in P. lineatus and P. discolor, and three pairs of autosomes in A. lituratus.

The species with the greatest number of NORs generated the lowest intensities of fluorescent signals, reinforcing the idea of that during the chromosomal rearrangements occurring in bat karyotypes, the NORs tended to cluster in some species and meet in others, maintaining a balance between mechanisms that increase and those that reduce the number of rDNA sites (Baker et al., 1992).

The presence of fluorescent signals in spermatocytes in smaller numbers than the number of NORs is a result of pairing of homologous chromosomes to form bivalents. This promotes the approach of NORs and reduces by half the expected number of signals in the primary spermatocytes in zygotene and leptotene. However, a temporary separation seems to occur at the beginning of pachytene that does not extend to the end of pachytene, which allowed us to see in some cells the signals corresponding to NORs separately.

\section{CONCLUSIONS}

In this study, the analysis of the species $C$. perspicillata showed that despite the species showing NOR on the X-chromosome, the behavior of its nucleolus during meiotic division did not differ from that of other species that have autosomal NORs.

Likewise, to correlate the changes in location and number of NORs of the other three species with the nucleolar behavior during meiosis, we found that nucleolar disassembly showed similar characteristics in these species, even with the NORs located on morphologically different chromosomes and distinct chromosomal regions (interstitial or terminal regions). The results suggest that the location of rDNA loci in the genome does not appear to interfere with nucleologenesis, at least in the bat species analyzed.

This study, although based on the standard cytogenetic procedures, without the refinements of kinetic and immunocytochemical methods, is the first report of the dynamics of meiotic cells of Chiroptera.

\section{ACKNOWLEDGMENTS}

We are grateful to Dr. Shirlei Maria Recco-Pimentel for providing the probe and to Dr. Sonia Maria Oliani for the use of optical equipment. Research supported by FAPESP, CAPES and CNPq.

\section{REFERENCES}

Abrams JM, White K, Fessler LI and Steller H (1993). Programmed cell death during Drosophila embryogenesis. Development 117: 29-43.

Andersen JS, Lam YW, Leung AK, Ong SE, et al. (2005). Nucleolar proteome dynamics. Nature 433: 77-83.

Baker R, Maltbie M, Owen J, Hamilton M, et al. (1992). Reduced number of ribosomal sites in bats: evidence for a 
mechanism to contain genome size. J. Mammal. 73: 847-858.

Boisvert FM, van Koningsbruggen, Navascues J and Lamond AI (2007). The multifunctional nucleolus. Nat. Rev. Mol. Cell Biol. 8: 574-585.

Carmo-Fonseca M, Mendes-Soares L and Campos I (2000). To be or not to be in the nucleolus. Nat. Cell Biol. 2: E107-E112.

Cattani MV and Papeschi AG (2004). Nucleolus organizing regions and semi-persistent nucleolus during meiosis in Spartocera fusca (Thunberg) (Coreidae, Heteroptera). Hereditas 140: 105-111.

Costa LC, Azeredo-Oliveira MTV and Tartarotti E (2008). Spermatogenesis and nucleolar activity in Triatoma klugi (Triatomine, Heteroptera). Genet. Mol. Biol. 31: 438-444.

Dimario PJ (2004). Cell and molecular biology of nucleolar assembly and disassembly. Int. Rev. Cytol. 239: 99-178.

Dundr M, Misteli T and Olson MO (2000). The dynamics of postmitotic reassembly of the nucleolus. J. Cell Biol. 150: 433-446.

Gall JG (2001). A role for Cajal bodies in assembly of the nuclear transcription machinery. FEBS Lett. 498: 164-167.

Hernandez-Verdun D (2005). Nucleolus in the spotlight. Cell Cycle 4: 106-108.

Hernandez-Verdun D (2006a). Nucleolus: from structure to dynamics. Histochem. Cell Biol. 125: 127-137.

Hernandez-Verdun D (2006b). The nucleolus: a model for the organization of nuclear functions. Histochem. Cell Biol. 126: $135-148$.

Hofgärtner FJ, Schmid M, Krone W, Zenzes MT, et al. (1979). Pattern of activity of nucleolus organizers during spermatogenesis in mammals as analyzed by silver-staining. Chromosoma 71: 197-216.

Kavalco KF and Pazza R (2004). A rapid alternative technique for obtaining silver-positive patterns in chromosomes. Genet. Mol. Biol. 27: 196-198.

Kotaja N and Sassone-Corsi P (2007). The chromatoid body: a germ-cell-specific RNA-processing centre. Nat. Rev. Mol. Cell Biol. 8: 85-90.

Leung AK and Lamond AI (2003). The dynamics of the nucleolus. Crit. Rev. Eukaryot. Gene Expr. 13: 39-54.

Leung AK, Gerlich D, Miller G, Lyon C, et al. (2004). Quantitative kinetic analysis of nucleolar breakdown and reassembly during mitosis in live human cells. J. Cell Biol. 166: 787-800.

Louvet E, Junera HR, Le Panse S and Hernandez-Verdun D (2005). Dynamics and compartmentation of the nucleolar processing machinery. Exp. Cell Res. 304: 457-470.

Mitchell JR, Cheng J and Collins K (1999). A box H/ACA small nucleolar RNA-like domain at the human telomerase RNA 3' end. Mol. Cell Biol. 19: 567-576.

Morielle E and Varella-Garcia M (1988). Variability of nucleolus organizer regions in phyllostomid bats. Rev. Bras. Genet. 11: 853-871.

Morielle-Souza A and de Azeredo-Oliveira MT (2008). Study of the nucleolar cycle and ribosomal RNA distribution during meiosis in triatomines (Triatominae, Heteroptera). Micron 39: 1020-1026.

Morielle-Versute E, Varella-Garcia M and Taddei VA (1996). Karyotypic patterns of seven species of molossid bats (Molossidae, Chiroptera). Cytogenet. Cell Genet. 72: 26-33.

Olson MO and Dundr M (2005). The moving parts of the nucleolus. Histochem. Cell Biol. 123: 203-216.

Olson MO, Dundr M and Szebeni A (2000). The nucleolus: an old factory with unexpected capabilities. Trends Cell Biol. 10: $189-196$.

Paniagua R, Nistal M, Amat P and Rodriguez MC (1986). Ultrastructural observations on nucleoli and related structures during human spermatogenesis. Anat. Embryol. 174: 301-306.

Peruquetti RL, Assis IM, Taboga SR and de Azeredo-Oliveira MT (2008). Meiotic nucleolar cycle and chromatoid body formation during the rat (Rattus novergicus) and mouse (Mus musculus) spermiogenesis. Micron 39: 419-425.

Peruquetti RL, Taboga SR and de Azeredo-Oliveira MT (2010). Characterization of Mongolian gerbil chromatoid bodies and their correlation with nucleolar cycle during spermatogenesis. Reprod. Domest. Anim. 45: 399-406.

Politz JC, Yarovoi S, Kilroy SM, Gowda K, et al. (2000). Signal recognition particle components in the nucleolus. Proc. Natl. Acad. Sci. U. S. A. 97: 55-60.

Politz JC, Tuft RA and Pederson T (2003). Diffusion-based transport of nascent ribosomes in the nucleus. Mol. Biol. Cell 14: 4805-4812.

Raška I, Koberna K, Malinsky J, Fidlerova H, et al. (2004). The nucleolus and transcription of ribosomal genes. Biol. Cell 96: 579-594.

Raška I, Shaw PJ and Cmarko D (2006a). Structure and function of the nucleolus in the spotlight. Curr. Opin. Cell Biol. 18: 325-334.

Raška I, Shaw PJ and Cmarko D (2006b). New insights into nucleolar architecture and activity. Int. Rev. Cytol. 255: 177-235. Savino TM, Gebrane-Younes J, De Mey J, Sibarita JB, et al. (2001). Nucleolar assembly of the rRNA processing machinery 
in living cells. J. Cell Biol. 153: 1097-1110.

Schmid M, Löser C, Schmidtke J and Engel W (1982). Evolutionary conservation of a common pattern of activity of nucleolus organizers during spermatogenesis in vertebrates. Chromosoma 86: 149-179.

Sirri V, Urcuqui-Inchima S, Roussel P and Hernandez-Verdun D (2008). Nucleolus: the fascinating nuclear body. Histochem. Cell Biol. 129: 13-31.

Souza MJ and Araújo MCP (1990). Conservative pattern of the G-bands and diversity of C-banding patterns and NORs in Stenodermatinae (Chiroptera-Phyllostomatidae). Rev. Bras. Genet. 13: 255-268.

Viegas-Péquignot E (1992). In situ Hybridization to Chromosomes with Biotinylated Probes. In: In situ Hybridization: A Practical Approach (Willernson D, ed.). Oxford University Press and IRL Press, Oxford, 137-158.

Visintin R and Amon A (2000). The nucleolus: the magician's hat for cell cycle tricks. Curr. Opin. Cell Biol. 12: 752.

Volleth M (1987). Differences in the location of nucleolus organizer regions in European vespertilionid bats. Cytogenet. Cell Genet. 44: 186-197. 